\title{
vWF/ADAMTS13 is associated with on-aspirin residual platelet reactivity and clinical outcome in patients with stable coronary artery disease
}

Ellen M. K. Warlo ${ }^{1,2,3^{*}}$ D, Alf-Åge R. Pettersen ${ }^{1,3,4}$, Harald Arnesen ${ }^{1,2,3}$ and Ingebjørg Seljeflot ${ }^{1,2,3}$

\begin{abstract}
Background: The mechanisms behind residual platelet reactivity (RPR) despite aspirin treatment are not established. It has been shown that coronary artery disease (CAD) patients with high on-aspirin RPR have elevated levels of von Willebrand factor (VWF). ADAMTS13 is a metalloprotease cleaving ultra large WWF multimers into less active fragments. Our aim was to investigate whether ADAMTS13 and VWF/ADAMTS13 ratio were associated with high RPR, and further with clinical endpoints after 2 years.

Methods: Stable aspirin-treated CAD patients $(n=999)$ from the ASCET trial. RPR was assessed by PFA-100. ADAMTS13 antigen and activity were analysed using chromogenic assays. Endpoints were a composite of acute myocardial infarction, stroke and death.

Results: The number of patients with high RPR was 258 (25.8\%). Their serum thromboxane $B_{2}\left(T \times B_{2}\right)$ levels were low, indicating inhibition of COX-1. They had significantly lower levels of ADAMTS13 antigen compared to patients with low RPR (517 vs $544 \mathrm{ng} / \mathrm{mL}, p=0.001$ ) and significantly lower ADAMTS13 activity (0.99 vs $1.04 \mathrm{IU} / \mathrm{mL}, p=0.020$ ). The differences were more pronounced when relating RPR to ratios of VWF/ADAMTS13 antigen and VWF/ADAMTS13 activity $(p<0.001$, both). We found an inverse correlation between VWF and ADAMTS13 antigen $(r=-0.14, p<0.001)$ and ADAMTS13 activity $(r=-0.11, \mathrm{p}<0.001)$. No correlations between $\mathrm{TXB}_{2}$ and ADAMTS13 antigen or activity, were observed, implying that ADAMTS13 is not involved in TXB2 production. Patients who experienced endpoints ( $n=73)$ had higher vWF level (113 vs $105 \%, p=0.032)$ and VWF/ADAMTS13 antigen ratio (0.23 vs 0.20, $p=0.012)$ compared to patients without. When dichotomizing VWF/ADAMTS13 antigen at median level we observed that patients above median had higher risk for suffering endpoints, with an adjusted OR of 1.86 ( $95 \% \mathrm{Cl} 1.45,2.82)$.
\end{abstract}

Conclusion: These results indicate that ADAMTS13 is of importance for RPR, and that it in combination with vWF also is associated with clinical endpoints in stable CAD patients on aspirin.

Trial registration: Clinicaltrials.gov NCT00222261. Registered 13.09.2005. Retrospectively registered.

Keywords: ADAMTS13, Von Willebrand factor, Aspirin, Residual platelet reactivity, Cardiovascular disease

\footnotetext{
* Correspondence: e.m.k.warlo@studmed.uio.no

${ }^{1}$ Center for Clinical Heart Research, Department of Cardiology, Oslo

University Hospital, Ullevaal, Pb 4956 Nydalen, 0424 Oslo, Norway

${ }^{2}$ Faculty of Medicine, University of Oslo, Oslo, Norway

Full list of author information is available at the end of the article
} 


\section{Background}

Von Willebrand factor (vWF) is an established marker of endothelial activation, and patients with elevated plasma levels of the ultra large vWF molecules are at high risk for future cardiovascular events [1-4].

vWF is a glycoprotein involved in both platelet activation and aggregation through its binding sites for GpIb and GpIIb/IIIa, respectively [5]. It is released to the circulation as ultra-large multimers from Weibel-Palade bodies in the endothelial cells, as well as from $\alpha$-granules in platelets. vWF occurs in different lengths in the circulation and its thrombotic properties differ depending on its size [6]. It is involved in haemostasis when released as ultra-large multimers, and the activity decreases as the size abates [5]. vWF's activity is also enhanced by shear stress, promoting conformational changes of the molecule to expose important binding sites [6]. vWF is cleaved into smaller fragments with reduced thrombotic activity by a metalloproteinase, ADAMTS13, "A Disintegrin And Metalloprotease with TromboSpondin type 1 motif, member 13" [7]. Reduced amount and activity of ADAMTS13 lead to less fragmentation of vWF with subsequent higher amount of the ultra-large vWF molecules, thus potentially a more prothrombotic state.

The role of ADAMTS13 in coronary artery disease (CAD) has not been established, and inconclusive results have been reported [3]. A recent meta-analysis showed low ADAMTS13 levels, both amount and activity, with concomitant ultra-large vWF, to be a risk factor for myocardial infarction [8]. The importance of ADAMTS13 for platelet reactivity, indirectly by acting on vWF or directly, is limited described.

Aspirin is a cornerstone in treatment of cardiovascular disease (CVD), but patients on aspirin may still experience new cardiovascular events. The phenomenon of residual platelet reactivity (RPR) despite use of aspirin has been extensively studied with diverging results [9]. The prevalence of high RPR and any predictive value vary considerably depending on the laboratory methods used [10, 11]. Thus, there is no recommendation to introduce platelet function testing in clinical practice. Regardless of this, the mechanisms behind high RPR assessed by platelet function testing are still not known in details. In the Aspirin Nonresponsiveness and Clopidogrel Endpoint Trial (ASCET) [12], it was shown that patients with high on-aspirin RPR had significantly elevated levels of vWF compared to patients with low RPR [13].

The aim of the present study was to investigate whether ADAMTS13 alone and as a vWF/ADAMTS13 ratio were associated with the presence of high $R P R$ in aspirin treated CAD patients. We hypothesised that low ADAMTS13 would contribute to high RPR. We further explored whether ADAMTS13 and the vWF/ ADAMTS13 ratio were associated with disease entities and risk factors and further with clinical outcome after 2 years follow-up.

\section{Methods \\ Study population}

This is a substudy of the ASCET trial which was performed at Center for Clinical Heart Research, Oslo University Hospital, Ullevaal, Oslo, Norway, from March 2003 until July 2010 [12]. The design of the trial has previously been published [14], and it is registered at https://www.clinicaltrials.gov/ (identification No. NCT00222261).

Patients $(n=1001)$ with stable CAD, previously verified by angiography, were included. All patients were on single antiplatelet therapy with aspirin $160 \mathrm{mg} / \mathrm{d}$ for at least 1 week prior to inclusion. Patients were randomized to either continue on aspirin $160 \mathrm{mg} / \mathrm{d}$ or change to clopidogrel $75 \mathrm{mg} / \mathrm{d}$ and were followed for 2 years for clinical endpoints. Patients in need of dual antiplatelet therapy or warfarin were excluded. The ASCET study was approved by the regional ethics committee and all patients gave their written consent.

Current smokers were defined as patients still smoking or former smokers who had quit less than 3 months ago. Hypertensives included patients with treated hypertension. The criteria for diabetes were patients with treated T1DM and T2DM or fasting blood glucose $>7 \mathrm{mmol} / \mathrm{L}$.

Clinical endpoints in the main ASCET study included unstable angina pectoris, myocardial infarction (MI), nonhaemorrhagic stroke and death. For the purpose of the present investigation we excluded the group of unstable angina pectoris as this is a less defined diagnosis. Thus, the recorded endpoints were $\mathrm{MI}$, non-haemorrhagic stroke and death. Endpoints were recorded at study visits and on request for patients unable to attend the final visit. An endpoint committee performed the evaluation of endpoints and internationally accepted diagnostic criteria were used.

\section{Blood sampling}

Blood samples were drawn at inclusion in fasting condition between 08.00 and $10.30 \mathrm{AM}, 24 \mathrm{~h}$ after last intake of aspirin. Citrated plasma (0.129 $\mathrm{M}$ in dilution 1:10) was stored on ice and separated within $30 \mathrm{~min}$ by centrifugation at $4{ }^{\circ} \mathrm{C}$ and $3000 \mathrm{~g}$ for $20 \mathrm{~min}$, and stored at $-80{ }^{\circ} \mathrm{C}$ until analysed for ADAMTS13 antigen and activity, performed by commercial methods (IMUBIND ${ }^{\circ}$ (Seksui Diagnostics GmbH, Pfungstadt, Germany and TECHNOZYM ${ }^{\circ}$ ADAMTS-13 activity assay, Technoclone, Vienna, Austria, respectively). We have previously reported on vWF, determined in citrated plasma by use of Asserachrom vWF Ag (Stago Diagnostica, Asnieres, France), and Thromboxane $B_{2}$, analysed in serum prepared from whole blood without anticoagulants and kept at $37^{\circ}$ for $1 \mathrm{~h}$ before centrifugation at $2500 \times \mathrm{g}$ for $10 \mathrm{~min}$ (Amersham Thromboxane $\mathrm{B}_{2}$ Biotrak Assay, GE Healthcare, Buckinghamshire, UK) $[12,13]$. 
Residual platelet reactivity (RPR)

The method has been described in details previously [12]. Briefly, citrated whole blood (0.129 M in dilution 1:10) was analysed within $2 \mathrm{~h}$ by the PFA100 system (Siemens Healthcare Diagnostics, Germany). This system stimulates platelet-based haemostasis in vitro by use of cartridges with collagen and epinephrine. Closure time (CT) was recorded to determine platelet function. The cut-off value, determined by testing 200 CAD patients not on antiplatelet therapy, was set at $196 \mathrm{~s}$ based on the 95 percentile in this cohort [15]. Patients with CT below this level were classified with high RPR and patients above this level with low RPR.

\section{Statistical analysis}

Continuous variables are presented as mean \pm SD or median (25th, 75th percentiles) when appropriate. Categorical data are presented as numbers or percentages. Students unpaired t-test or Mann-Whitney U test were used to compare continuous variables between groups, and group comparisons for categorical variables were performed by Pearson's chisquared test. Correlation analyses were performed by Spearman's rho. Logistic regression analysis was used to adjust for relevant covariates, estimated from Additional file 1 Table S1. A $p$-value $<5 \%$ was considered statistically significant. SPSS version 22 (SPSS Inc., IL, USA) was used.

\section{Results}

Baseline characteristics of the total population and according to the presence of low or high RPR are shown in Table 1. Blood samples from two patients were not available for ADAMTS13 analyses, thus all results are given for 999 patients. The number of patients with high RPR was 258 (25.8\%). $\mathrm{TxB}_{2}$ levels were low in all patients, compared to levels in individuals not on aspirin [15]. There was a significantly lower percentage of hypertensives, and a higher

Table 1 Baseline characteristics of the total populating and according to the presence of low or high RPR

\begin{tabular}{|c|c|c|c|c|}
\hline & Total population & Low RPR $(n=741)$ & High RPR $(n=258)$ & $p$-value \\
\hline Age (years) ${ }^{a}$ & $62.4(36-81)$ & $62.6(36-81)$ & $61.7(41-80)$ & 0.154 \\
\hline Sex, female, n (\%) & $218(21.8)$ & $159(21.5)$ & $58(22.5)$ & 0.731 \\
\hline Caucasian, n (\%) & $968(96.8)$ & $724(97.7)$ & $243(94.2)$ & 0.006 \\
\hline \multicolumn{5}{|l|}{ Cardiovascular risk factors, n (\%) } \\
\hline Current smoking & $203(20.3)$ & $136(18.4)$ & $67(26.0)$ & 0.009 \\
\hline Hypertension & $556(55.7)$ & $433(58.4)$ & $123(47.9)$ & 0.003 \\
\hline Diabetes mellitus & $200(20.0)$ & $148(20.0)$ & $52(20.2)$ & 0.950 \\
\hline Previous CVD ${ }^{d}$ & $635(63.6)$ & $462(62.3)$ & $172(66.9)$ & 0.189 \\
\hline - Previous myocardial infarction & $436(43.7)$ & $314(42.4)$ & $121(47.1)$ & 0.195 \\
\hline Systolic blood pressure $(\mathrm{mmHg})^{b}$ & $140 \pm 19$ & $141 \pm 19$ & $136 \pm 19$ & $<0.001$ \\
\hline Diastolic blood pressure $(\mathrm{mmHg})^{\mathrm{b}}$ & $82 \pm 10$ & $82 \pm 10$ & $81 \pm 9$ & 0.040 \\
\hline Body mass index $\left(\mathrm{kg} / \mathrm{m}^{2}\right)^{\mathrm{b}}$ & $27.4 \pm 3.7$ & $27.5 \pm 3.8$ & $27.2 \pm 3.5$ & 0.237 \\
\hline \multicolumn{5}{|l|}{ Blood tests } \\
\hline Total cholesterol $(\mathrm{mmol} / \mathrm{L})^{\mathrm{b}}$ & $4.55 \pm 0.98$ & $4.55 \pm 0.99$ & $4.53 \pm 0.96$ & 0.793 \\
\hline LDL cholesterol $(\mathrm{mmol} / \mathrm{L})^{\mathrm{b}}$ & $2.53 \pm 0.83$ & $2.53 \pm 0.83$ & $2.53 \pm 0.83$ & 0.998 \\
\hline HDL cholesterol $(\mathrm{mmol} / \mathrm{L})^{\mathrm{b}}$ & $1.33 \pm 0.41$ & $1.34 \pm 0.42$ & $1.31 \pm 0.37$ & 0.277 \\
\hline Triglycerides $(\mathrm{mmol} / \mathrm{L})^{c}$ & $1.31(0.93,1.84)$ & $1.29(0.91,1.84)$ & $1.35(0.97,1.85)$ & 0.144 \\
\hline Residual platelet reactivity, n (\%) & $258(25.8)$ & & & \\
\hline $\mathrm{TxB}_{2}(\mathrm{ng} / \mathrm{mL})^{\mathrm{a}}$ & $2.7(0-21)$ & $2.6(0-21)$ & $3.0(0-15)$ & 0.102 \\
\hline \multicolumn{5}{|l|}{ Medication, n (\%) } \\
\hline Statins & $982(98.3)$ & $727(98.2)$ & $254(98.4)$ & 0.825 \\
\hline B-blockers & $755(75.8)$ & $561(76.1)$ & $193(74.8)$ & 0.672 \\
\hline Calcium channel blockers & $255(25.6)$ & $196(26.5)$ & $59(23.0)$ & 0.272 \\
\hline ACE-inhibitors & $263(26.5)$ & $200(27.1)$ & $63(24.7)$ & 0.455 \\
\hline ARB & $239(24.0)$ & $187(25.3)$ & $52(20.4)$ & 0.111 \\
\hline
\end{tabular}

$C V D$ cardiovascular disease, $M I$ myocardial infarction, $P C l$ percutaneous coronary intervention, $C A B G$ coronary artery bypass graft, $T x B_{2}$ thromboxane $B_{2}, A C E$ angiotensin-converting enzyme, $A R B$ angiotensin II receptor blockers

${ }^{\varsigma} p$-values refer to differences between the groups with high or low RPR

${ }^{\mathrm{a}}$ mean (range) ${ }^{\mathrm{b}}$ mean $\pm \mathrm{SD}{ }^{\mathrm{c}}$ median (25th, 75th percentiles) ${ }^{\mathrm{d}}$ Previous $\mathrm{MI}, \mathrm{PCl}, \mathrm{CABG}$, non-haemorrhagic stroke

Significant $\mathrm{p}$-values are highlighted with boldface 
Table 2 Baseline levels of the measured markers in the total population and according to the presence of low or high RPR

\begin{tabular}{lllll}
\hline & Total population & Low RPR $(n=741)$ & High RPR $(n=258)$ & $p$-value \\
\hline VWF $(\%)$ & $105(82,133)$ & $100(79,126)$ & $124(94,145)$ & $511(448,580)$ \\
ADAMTS13 antigen $(\mathrm{ng} / \mathrm{mL})$ & $532(461,606)$ & $537(469,613)$ & $0.99(0.76,1.16)$ & $\mathbf{0 . 0 0 1}$ \\
ADAMTS13 activity $(\mathrm{IU} / \mathrm{mL})$ & $1.03(0.83,1.19)$ & $1.04(0.84,1.19)$ & $0.23(0.18,0.31)$ & $\mathbf{0 . 0 2 0}$ \\
Ratio VWF/ADAMTS13 antigen & $0.20(0.15,0.26)$ & $0.19(0.14,0.25)$ & $127(93,192)$ & $<0.001$ \\
Ratio VWF/ADAMTS13 activity & $107(77,152)$ & $101(73,137)$ & $236(200,273)$ & $\mathbf{0 . 0 0 1}$ \\
Platelet count $\left(\times 10^{9} / \mathrm{L}\right)$ & $227(195,264)$ & $224(192,261)$ & $\mathbf{0 . 0 1 0}$
\end{tabular}

Values are given as median (25th, 75th percentiles)

${ }_{\text {s }}$-values refer to differences between the groups with high or low RPR

Significant $\mathrm{p}$-values are highlighted with boldface

proportion of smokers in the high RPR group compared to the group with low RPR. There was no significant difference in clinical endpoints with regard to high and low RPR. These data on patients with high and low RPR have previously been published [12, 13].

\section{ADAMTS13 as related to RPR}

Levels of ADAMTS13 antigen and activity are shown in Table 2, in the total cohort and according to the RPR status. Patients with high RPR had significantly higher levels of vWF $(p<0.001)$ and platelet count $(p=0.010)$ compared to those with low RPR, as previously published [13]. In the present investigation we observed that patients with high RPR had significantly lower levels of both ADAMTS13 antigen and activity compared to individuals with low RPR
( $p=0.001, p=0.020$, respectively). When calculating ratios of vWF/ADAMTS13 antigen and vWF/ADAMTS13 activity the differences were even more pronounced, showing both ratios to be significantly higher in patients with high RPR ( $p<0.001$ for both).

Statistically significant, but weak inverse correlations were found between the levels of vWF and ADAMTS13 antigen $(r=-0.14, p<0.001)$ and ADAMTS13 activity $(r=-0.11, \mathrm{p}<0.001)$. ADAMTS13 antigen and activity were significantly inter-correlated $(r=0.47, \mathrm{p}<0.001)$.

\section{ADAMTS13 as related to disease entities}

Table 3 shows ADAMTS13 antigen and activity as related to different cardiovascular risk factors. When dichotomizing age at median level, we found that patients

Table 3 Baseline levels of the measured markers according to different clinical conditions

\begin{tabular}{|c|c|c|c|c|c|c|c|}
\hline & & $n$ & VWF (\%) & ADAMTS13 antigen (ng/mL) & ADAMTS13 activity (IU/mL) & Ratio antigen & Ratio activity \\
\hline \multirow[t]{3}{*}{ Age $>$ median (62 years) } & + & 500 & $110(88,137)$ & $519(455,581)$ & $1.00(0.79,1.15)$ & $0.21(0.17,0.29)$ & $117(86,170)$ \\
\hline & - & 500 & $100(79,127)$ & $547(467,627)$ & $1.05(0.90,1.21)$ & $0.19(0.14,0.25)$ & $97(70,136)$ \\
\hline & $p$ & & $<0.001$ & $<0.001$ & $<0.001$ & $<0.001$ & $<0.001$ \\
\hline \multirow[t]{3}{*}{ Sex (female) } & + & 218 & $106(82,136)$ & $549(463,622)$ & $1.04(0.84,1.19)$ & $0.20(0.14,0.26)$ & $111(76,157)$ \\
\hline & - & 782 & $106(83,133)$ & $525(460,596)$ & $1.02(0.82,1.19)$ & $0.20(0.15,0.27)$ & $106(77,150)$ \\
\hline & $\mathrm{p}$ & & 0.840 & 0.052 & 0.877 & 0.611 & 0.726 \\
\hline \multirow[t]{3}{*}{ Diabetes } & + & 200 & $111(83,141)$ & $557(465,639)$ & $1.05(0.81,1.18)$ & $0.20(0.15,0.27)$ & $117(84,170)$ \\
\hline & - & 800 & $105(83,131)$ & $524(459,596)$ & $1.02(0.83,1.19)$ & $0.20(0.15,0.26)$ & $106(76,147)$ \\
\hline & $\mathrm{p}$ & & 0.023 & 0.003 & 0.931 & 0.602 & 0.059 \\
\hline \multirow[t]{3}{*}{ Hypertension } & + & 556 & $104(80,132)$ & $530(463,606)$ & $1.01(0.79,1.16)$ & $0.20(0.15,0.26)$ & $107(76,150)$ \\
\hline & - & 443 & $108(86,134)$ & $533(458,607)$ & $1.05(0.86,1.21)$ & $0.20(0.16,0.28)$ & $106(77,153)$ \\
\hline & $p$ & & 0.033 & 0.959 & 0.034 & 0.081 & 0.962 \\
\hline \multirow[t]{3}{*}{ Previous CVD } & + & 635 & $110(86,137)$ & $534(463,608)$ & $1.02(0.82,1.19)$ & $0.20(0.16,0.27)$ & $110(82,160)$ \\
\hline & - & 364 & $98(78,126)$ & $525(456,602)$ & $1.04(0.85,1.18)$ & $0.19(0.14,0.25)$ & $103(72,136)$ \\
\hline & $\mathrm{p}$ & & $<0.001$ & 0.453 & 0.663 & 0.001 & 0.002 \\
\hline \multirow[t]{3}{*}{ Smokers } & + & 203 & $107(88,134)$ & $520(441,590)$ & $1.03(0.86,1.19)$ & $0.20(0.16,0.28)$ & $108(78,151)$ \\
\hline & - & 796 & $105(83,133)$ & $532(466,607)$ & $1.02(0.82,1.18)$ & $0.20(0.15,0.26)$ & $107(77,152)$ \\
\hline & $p$ & & 0.621 & 0.100 & 0.306 & 0.515 & 0.796 \\
\hline
\end{tabular}

Values are given as median (25th, 75th percentiles)

$p$-values refer to differences in the measured marker between patients having the specified clinical condition or not

Significant $\mathrm{p}$-values are highlighted with boldface 
above median (62 years) had higher levels of vWF, lower ADAMTS13 antigen and activity, and higher ratios $(p<0.001$, for all). No sex differences were observed in the measured markers. Diabetic patients had significantly higher levels of both vWF $(p=0.023)$ and ADAMTS13 antigen $(p=0.003)$, whereas hypertensive patients had significantly lower levels of vWF $(p=0.033)$ and lower ADAMTS13 activity $(p=0.034)$ compared to normotensives. Patients with previous CVD had significantly higher levels of vWF $(\mathrm{p}<0.001)$ and also higher vWF/ADAMTS13, both antigen ratio $(p=0.001)$ and activity ratio $(p=0.002)$. We observed no differences between smokers and non-smokers.

\section{ADAMTS13 as related to clinical endpoints}

After 2 years follow-up 73 clinical endpoints (MI, stroke or death) were recorded. These patients had higher levels of vWF and vWF/ADAMTS13 antigen ratio at inclusion compared to patients without endpoints (Table 4). We found no significant differences in the levels of ADAMTS13, neither antigen nor activity.

When dividing vWF and vWF/ADAMTS13 antigen ratio levels into quartiles, there were no significant trends for frequency of endpoints ( $p=0.135, p=0.071$, respectively) (Fig. 1). Nevertheless, we observed a potential cut-off level at the lowest quartile for vWF (Fig 1a). When dichotomizing vWF at this level, a higher event rate was demonstrated in patients with levels in the three upper quartiles compared to those in the lowest quartile with an OR of 2.18 (95\% CI 1.10, 4.31). The significance was, however, lost after adjusting for the covariates age, sex, diabetes, and previous CVD $(p=0.077)$.

When dichotomizing vWF/ADAMTS13 antigen at the median value (Fig 1b), an OR of 1.89 (95\% CI 1.15, 3.11) for suffering an event when having levels above the median was observed $(p=0.011)$. After adjustments for covariates the OR was $1.68(95 \% \mathrm{CI} 1.01,2.80)$ and the significance was retained $(p=0.045)$. Additional adjustment for the randomized treatment principle did not influence the results.

\section{Discussion}

This study was performed in a stable CAD population where 258 (25.8\%) patients had high RPR, determined by the PFA- 100 method. $\mathrm{TxB}_{2}$ levels were low, indicating adequate aspirin compliance. High RPR patients had higher levels of vWF compared to patients with low RPR as previously published [13].

Our main findings were that patients with high RPR had significantly lower levels of ADAMTS13, both antigen and activity, and higher vWF/ADAMTS13 ratios, and further significantly higher vWF levels and vWF/ADAMTS13 antigen ratio in patients who experienced a clinical endpoint after 2 years compared to patients who did not.

To our knowledge, this is the first study reporting on the importance of ADAMTS13 on RPR in stable aspirintreated CAD patients. Our results are mostly in line with a similar study by Marcucci et al. using other methods for RPR in a smaller CAD population, however, performed in the acute phase [16]. They observed significantly higher vWF and lower ADAMTS13 activity, but no difference in ADAMTS13 antigen in patients with high RPR. The different results on ADAMTS13 antigen might be due to different population sizes, but also the acute phase with a high degree of inflammation, which is known to affect both vWF and ADAMTS13 levels [17].

In our study RPR was related to both the amount and the activity of ADAMTS13. A possible mechanism is that low ADAMTS13 levels cause less fragmentation of vWF, resulting in more long vWF molecules that can activate platelets, thereby a higher RPR. We do not know whether high vWF levels per se cause high RPR or only act as a covariate. In the present situation the association between vWF and ADAMTS13 may be due to the reciprocal course of these molecules, i.e. high vWF may lead to low ADAMTS13 as a result of the increased availability of vWF which may lead to an "exhaustion" or elimination of ADAMTS13 by unknown mechanisms [17].

We observed only a weak correlation between vWF and ADAMTS13 which is in accordance with other reports, showing either weak or no correlation [18-20]. Even though it is well established that ADAMTS13

Table 4 Baseline levels of the measured markers according to clinical endpoint or no endpoint

\begin{tabular}{llll}
\hline & Endpoint $(n=73)$ & No endpoint $(n=927)$ & $P$-value \\
\hline VWF, \% & $113(93,141)$ & $105(81,133)$ & $\mathbf{0 . 0 3 2}$ \\
ADAMTS-13 antigen, $\mathrm{ng} / \mathrm{mL}$ & $506(452,576)$ & $533(462,607)$ & 0.147 \\
ADAMTS-13 activity, IU/mL & $1.00(0.73,1.13)$ & $1.03(0.83,1.19)$ & 0.212 \\
Ratio antigen & $0.23(0.17,0.29)$ & $0.20(0.15,0.26)$ & $\mathbf{0 . 0 1 2}$ \\
Ratio activity & $119(88,175)$ & $107(77,150)$ & 0.051 \\
Platelet count $\left(\times 10^{9} / \mathrm{L}\right)$ & $221(181,272)$ & $228(196,263)$ & 0.446 \\
\hline
\end{tabular}

Values are given as median (25th, 75th percentiles)

${ }^{\S} p$-values refer to differences between the groups with high or low RPR

Significant $\mathrm{p}$-values are highlighted with boldface 

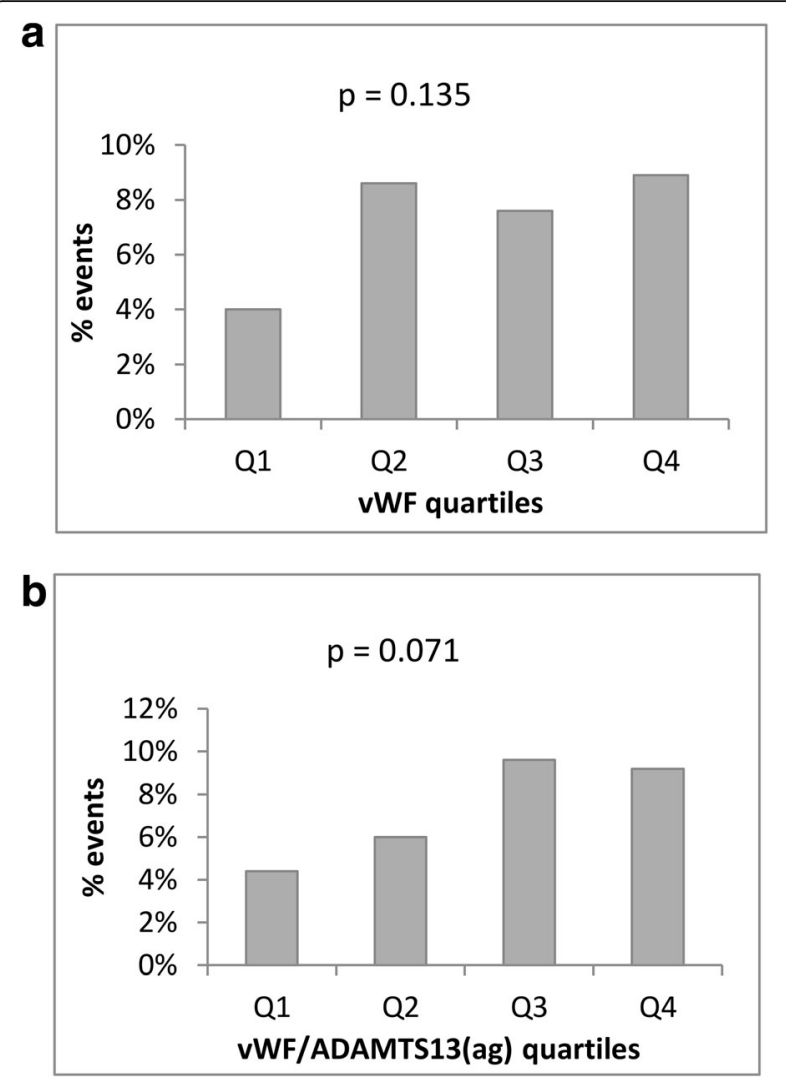

Fig. 1 a Endpoints during 2 years follow-up according to quartiles of VWF. $p$-value refers to trend analysis. $\mathbf{b}$ Endpoints during 2 years follow-up according to quartiles of VWF/ADAMTS13 antigen ratio. $p$-value refers to trend analysis

cleaves vWF, both are affected by other factors that might weaken this correlation [21-23]. It has also been suggested that the two molecules, independent of each other, are associated with clinical endpoints, and that ADAMTS13 have cardioprotective properties beyond vWF cleavage [20, 24, 25].

Both vWF and ADAMTS13, as well as the ratios were significantly associated with age, in line with other reports [26-30]. No significant sex differences were observed. Lower ADAMTS13, both antigen and activity have previously been shown in men [27-30]. Higher levels of both vWF and ADAMTS13 antigen were observed in diabetic patients. High vWF have generally been associated with diabetes, but the results for ADAMTS13 are more diverging [20, 29-31]. In the Rotterdam study higher ADAMTS13 activity in patients with diabetes compared to those without was reported [29], and they also showed ADAMTS13 activity to be an independent risk factor for both prediabetes and diabetes type 2 [32]. In hypertensive patients higher vWF and lower ADAMTS13 activity were found, which to some degree is in accordance with other reports, although varying results exist [26, 31]. Also, patients with previous CVD had higher vWF levels in our study. There are some case-control studies supporting this result, whereas the observations on ADAMTS13 are diverging $[19,20]$. We found no differences between smokers and non-smokers which is in accordance with previous reports [20,31].

Our observations of vWF and ADAMTS13 to be associated with clinical endpoints are partly in accordance with previous reports. vWF have repeatedly been associated with increased risk of coronary heart disease [3]. We observed higher levels of vWF in patients experiencing clinical endpoints, however, the significance was lost when adjusting for covariates. This might strengthen the recent suggestion that vWF is more modestly associated with coronary heart disease than previously estimated [32].

Previous reports on ADAMTS13, both antigen and activity as related to clinical outcome are inconclusive, and we could not demonstrate any association between ADAMTS13 and clinical endpoints. The meta-analysis by Maino et al. concluded that ADAMTS13 levels below the 5th percentile was associated with increased risk of MI [8], whereas Sonneveld et al. concluded an uncertainty on whether ADAMTS13 increases the risk of arterial thrombosis due to diverging results and lack of prospective studies [3].

We could, however, demonstrate that patients who suffered a clinical endpoint had significantly higher levels of vWF/ADAMTS13 antigen ratio. Patients with ratio above median level had an OR of 1.68 for suffering an event, significant also after adjustments for relevant covariates, indicating that the combination of vWF and ADAMTS13 antigen might be a better prognostic marker than vWF alone. To the best of our knowledge this is the first report on vWF/ADAMTS13 antigen ratio in relation to future cardiovascular events.

\section{Study limitations}

The platelet function test used in this study, PFA-100 with collagen and epinephrine cartridges, has been questioned with regard to response to aspirin. It is a COX-1-non-specific test, and therefore not quite suitable to detect real aspirin-resistance. High RPR by this method is not necessarily due to insufficient inhibition of COX-1, but might be caused by platelet activation through other pathways. Although high RPR by the PFA-100 method have, in some studies shown to be associated with increased risk of clinical endpoints [11], in our population, as well as in other studies, PFA-100 was not able to identify these high-risk patients [12, 33, 34]. RPR was determined only at baseline without re-testing during the follow-up period. It should also be emphasized that the method is dependent of vWF levels $[13,35]$. However, in our population very few had levels below or above the reference values. vWF varies with blood type and the coagulation factors VIII and 
fibrinogen concentrations [21, 36, 37]. It is also uncertain whether the vWF antigen levels measured reflect vWF length.

\section{Conclusion}

In our population of stable CAD patients on aspirin treatment, low ADAMTS13 levels were associated with high RPR and in combination with VWF associated with clinical outcome after 2 years. Beyond the properties of ADAMTS13 to cleave vWF, other mechanisms behind these associations are not clear and further investigations are needed.

\section{Additional file}

Additional file 1: Table S1. Baseline characteristics according to clinical endpoint or no endpoint. (PDF 173 kb)

\section{Abbreviations}

ACE: Angiotensin-converting enzyme; ADAMTS13: A Disintegrin And Metalloprotease with TromboSpondin type 1 motif, member 13; ARB: Angiotensin II receptor blockers; ASCET: Aspirin Nonresponsiveness and Clopidogrel Endpoint Trial; CABG: Coronary artery bypass graft;

CAD: Coronary artery disease; CT: Closure time; CVD: Cardiovascular disease; MI: Myocardial infarction; PCl: Percutaneous coronary intervention; RPR: Residual platelet reactivity; $\mathrm{TxB}_{2}$ : Thromboxane $\mathrm{B}_{2}$; vWF: Von Willebrand Factor

\section{Acknowledgements}

The authors would like to thank medical laboratory technologist Sissel Åkra for excellent technical assistance.

\section{Funding}

The study was financially supported by The Research Council of Norway through the Medical Student Research Program at the University of Oslo, specified to the first author, and Stein Erik Hagens Foundation for Clinical Heart Research, Oslo Norway by unrestricted grants to the research milieu (recipients $\mathrm{HA}$ and IS).

\section{Availability of data and materials}

The analyses performed for the current study are not publicly available, but are available from the corresponding author on reasonable request.

\section{Authors' contributions}

EW performed laboratory and statistically analyses, contributed to interpretation of results and drafting the manuscript. AP was the principa investigator in the ASCET study, involved in planning of the study, the recruitment and follow-up of the study participants and acquisition of clinical data. IS and HA contributed to the overall design of the study, the interpretation of the results and the intellectual content of the manuscript. All authors read and approved the final manuscript.

\section{Ethics approval and consent to participate}

The ASCET study was approved by the Regional Committee for Medical Research Ethics, Health East with the ID-number: 652, conducted in accordance with the ethical principles of the Declaration of Helsinki. All patients gave their written informed consent to participate.

\section{Consent for publication}

Not applicable

\section{Competing interests}

The authors declare that they have no competing interest.

\section{Publisher's Note}

Springer Nature remains neutral with regard to jurisdictional claims in published maps and institutional affiliations.

\section{Author details}

${ }^{1}$ Center for Clinical Heart Research, Department of Cardiology, Oslo University Hospital, Ullevaal, Pb 4956 Nydalen, 0424 Oslo, Norway. ${ }^{2}$ Faculty of Medicine, University of Oslo, Oslo, Norway. ${ }^{3}$ Center for Heart Failure Research, University of Oslo, Oslo, Norway. ${ }^{4}$ Department of Medicine, Vestre Viken HF, Ringerike Hospital, Hønefoss, Norway.

Received: 2 October 2017 Accepted: 9 November 2017

Published online: 22 November 2017

\section{References}

1. Morange PE, Simon C, Alessi MC, LuC G, Arveiler D, Ferrieres J, et al. Endothelial cell markers and the risk of coronary heart disease: the prospective epidemiological study of myocardial infarction (PRIME) study. Circulation. 2004;109:1343-8. https://doi.org/10.1161/01.cir. 0000120705.55512.ec.

2. Thompson SG, Kienast J, Pyke SD, Haverkate F, van de Loo JC. Hemostatic factors and the risk of myocardial infarction or sudden death in patients with angina pectoris. European concerted action on thrombosis and disabilities angina pectoris study group. N Engl J Med. 1995;332:635-41. https://doi.org/10.1056/nejm199503093321003.

3. Sonneveld MA, de Maat MP, Leebeek FW. Von Willebrand factor and ADAMTS13 in arterial thrombosis: a systematic review and meta-analysis. Blood Rev. 2014;28:167-78. https://doi.org/10.1016/j.blre.2014.04.003.

4. Folsom AR, KK W, Shahar E, Davis CE. Association of hemostatic variables with prevalent cardiovascular disease and asymptomatic carotid artery atherosclerosis. The atherosclerosis risk in communities (ARIC) study investigators. Arterioscler Thromb. 1993;13:1829-36. http://atvb.ahajournals. org/content/atvbaha/13/12/1829.full.pdf

5. Stockschlaeder M, Schneppenheim R, Budde U. Update on von Willebrand factor multimers: focus on high-molecular-weight multimers and their role in hemostasis. Blood Coagul Fibrinolysis. 2014;25:206-16. https://doi.org/10. 1097/mbc.0000000000000065.

6. Reininger AJ. The function of ultra-large von Willebrand factor multimers in high shear flow controlled by ADAMTS13. Hamostaseologie. 2015;35:225-33. https://doi.org/10.5482/hamo-14-12-0077.

7. Eerenberg ES, Levi M. The potential therapeutic benefit of targeting ADAMTS13 activity. Semin Thromb Hemost. 2014;40:28-33. https://doi.org/ 10.1055/s-0033-1363156.

8. Maino A, Siegerink B, Lotta LA, Crawley JT, le Cessie S, Leebeek FW, et al. Plasma ADAMTS-13 levels and the risk of myocardial infarction: an individual patient data meta-analysis. J Thromb Haemost. 2015;13:1396-404. https://doi.org/10.1111/jth.13032.

9. Pettersen $A A$, Arnesen $H$, Seljeflot I. A brief review on high on-aspirin residual platelet reactivity. Vasc Pharmacol. 2015;67-69:6-9. https://doi.org/ 10.1016/j.vph.2015.03.018.

10. Hovens MM, Snoep JD, Eikenboom JC, van der Bom JG, Mertens BJ, Huisman MV. Prevalence of persistent platelet reactivity despite use of aspirin: a systematic review. Am Heart J. 2007;153:175-81. https://doi.org/10. 1016/j.ahj.2006.10.040

11. Wisman PP, Roest M, Asselbergs FW, de Groot PG, Moll FL, van der Graaf $Y$, et al. Platelet-reactivity tests identify patients at risk of secondary cardiovascular events: a systematic review and meta-analysis. J Thromb Haemost. 2014;12:736-47. https://doi.org/10.1111/jth.12538.

12. Pettersen AA, Seljeflot I, Abdelnoor M, Arnesen $\mathrm{H}$. High on-aspirin platelet reactivity and clinical outcome in patients with stable coronary artery disease: results from ASCET (aspirin nonresponsiveness and Clopidogrel endpoint trial). J Am Heart Assoc. 2012;1:e000703. https://doi.org/10.1161/jaha.112.000703.

13. Pettersen AA, Arnesen H, Opstad TB, Bratseth V, Seljeflot I. Markers of endothelial and platelet activation are associated with high on-aspirin platelet reactivity in patients with stable coronary artery disease. Thromb Res. 2012;130:424-8. https://doi.org/10.1016/j.thromres.2012.06.016.

14. Pettersen AA, Seljeflot I, Abdelnoor M, Arnesen H. Unstable angina, stroke, myocardial infarction and death in aspirin non-responders. A prospective, randomized trial. The ASCET (ASpirin non-responsiveness and Clopidogrel endpoint trial) design. Scand Cardiovasc J. 2004;38(6):353. https://doi.org/10. 1080/14017430410024324 
15. Andersen K, Hurlen M, Arnesen H, Seljeflot I. Aspirin non-responsiveness as measured by PFA-100 in patients with coronary artery disease. Thromb Res. 2002;108:37-42

16. Marcucci R, Cesari F, Cinotti S, Paniccia R, Gensini GF, Abbate R, et al. ADAMTS-13 activity in the presence of elevated von Willebrand factor levels as a novel mechanism of residual platelet reactivity in high risk coronary patients on antiplatelet treatment. Thromb Res. 2008;123:130-6. https://doi. org/10.1016/j.thromres.2008.05.017.

17. Reiter RA, Varadi K, Turecek PL, Jilma B, Knobl P. Changes in ADAMTS13 (von-Willebrand-factor-cleaving protease) activity after induced release of von Willebrand factor during acute systemic inflammation. Thromb Haemost. 2005;93:554-8. https://doi.org/10.1160/th04-08-0467.

18. Andersson HM, Siegerink B, Luken BM, Crawley JT, Algra A, Lane DA, et al. High WWF, low ADAMTS13, and oral contraceptives increase the risk of ischemic stroke and myocardial infarction in young women. Blood. 2012; 119:1555-60. https://doi.org/10.1182/blood-2011-09-380618.

19. Bongers TN, de Bruijne EL, Dippel DW, de Jong AJ, Deckers JW, Poldermans D, et al. Lower levels of ADAMTS13 are associated with cardiovascular disease in young patients. Atherosclerosis. 2009;207:250-4. https://doi.org/ 10.1016/j.atherosclerosis.2009.04.013.

20. Crawley JT, Lane DA, Woodward M, Rumley A, Lowe GD. Evidence that high von Willebrand factor and low ADAMTS-13 levels independently increase the risk of a non-fatal heart attack. J Thromb Haemost. 2008;6:583-8. https:// doi.org/10.1111/j.1538-7836.2008.02902.x.

21. Gallinaro L, Cattini MG, Sztukowska M, Padrini R, Sartorello F, Pontara E, et al. A shorter von Willebrand factor survival in O blood group subjects explains how $\mathrm{ABO}$ determinants influence plasma von Willebrand factor. Blood. 2008;111:3540-5. https://doi.org/10.1182/blood-2007-11-122945.

22. Zheng XL. Structure-function and regulation of ADAMTS-13 protease. J Thromb Haemost. 2013:11 Suppl 1:11-23. https://doi.org/10.1111/jth.12221.

23. Tersteeg C, Fijnheer R, Pasterkamp G, de Groot PG, Vanhoorelbeke K, de Maat S, et al. Keeping von Willebrand factor under control: alternatives for ADAMTS13. Semin Thromb Hemost. 2016;42:9-17. https://doi.org/10.1055/s-0035-1564838.

24. Feng Y, Li X, Xiao J, Li W, Liu J, Zeng X, et al. ADAMTS13: more than a regulator of thrombosis. Int J Hematol. 2016;104:534-9. https://doi.org/10. 1007/s12185-016-2091-2.

25. Chion CK, Doggen CJ, Crawley JT, Lane DA, Rosendaal FR. ADAMTS13 and von Willebrand factor and the risk of myocardial infarction in men. Blood. 2007;109:1998-2000. https://doi.org/10.1182/blood-2006-07-038166.

26. Enooku $K$, Kato $R$, Ikeda $H$, Kurano $M$, Kume $Y$, Yoshida $H$, et al. Inverse correlations between serum ADAMTS13 levels and systolic blood pressure, pulse pressure, and serum C-reactive protein levels observed at a general health examination in a Japanese population: a cross-sectional study. Clin Chim Acta. 2013;421:147-51. https://doi.org/10.1016/j.cca.2013.03.012.

27. Kokame K, Nobe Y, Kokubo Y, Okayama A, Miyata T. FRETS-WWF73, a first fluorogenic substrate for ADAMTS13 assay. Br J Haematol. 2005;129:93-100. https://doi.org/10.1111/j.1365-2141.2005.05420.x.

28. Kokame K, Sakata T, Kokubo Y, Miyata T. Von Willebrand factor-toADAMTS13 ratio increases with age in a Japanese population. J Thromb Haemost. 2011;9:1426-8. https://doi.org/10.1111/j.1538-7836.2011.04333.x.

29. Sonneveld MA, de Maat MP, Portegies ML, Kavousi M, Hofman A, Turecek PL, et al. Low ADAMTS13 activity is associated with an increased risk of ischemic stroke. Blood. 2015;126:2739-46. https://doi.org/10.1182/blood2015-05-643338

30. Skeppholm M, Kallner A, Kalani M, Jorneskog G, Blomback M, Wallen HN ADAMTS13 and von Willebrand factor concentrations in patients with diabetes mellitus. Blood Coagul Fibrinolysis. 2009;20:619-26. https://doi.org/ 10.1097/MBC.0b013e32832da183.

31. Miura M, Kaikita K, Matsukawa M, Soejima K, Fuchigami S, Miyazaki Y, et al. Prognostic value of plasma von Willebrand factor-cleaving protease (ADAMTS13) antigen levels in patients with coronary artery disease. Thromb Haemost. 2010;103:623-9. https://doi.org/10.1160/th09-08-0568.

32. de Vries PS, van Herpt TT, Ligthart S, Hofman A, Ikram MA, van Hoek M, et al. ADAMTS13 activity as a novel risk factor for incident type 2 diabetes mellitus: a population-based cohort study. Diabetologia. 2016. doi: https:// doi.org/10.1007/s00125-016-4139-5.

33. Pamukcu B, Oflaz H, Onur I, Oncul A, Ozcan M, Umman B, et al. Clinical relevance of aspirin resistance in patients with stable coronary artery disease: a prospective follow-up study (PROSPECTAR). Blood Coagul Fibrinolysis. 2007;18:187-92. https://doi.org/10.1097/MBC.0b013e328040c115.
34. Poulsen TS, Kristensen SR, Korsholm L, Haghfelt T, Jorgensen B, Licht PB, et al. Variation and importance of aspirin resistance in patients with known cardiovascular disease. Thromb Res. 2007;120:477-84. https://doi.org/10. 1016/j.thromres.2006.10.022.

35. Cattaneo M, Federici AB, Lecchi A, Agati B, Lombardi R, Stabile F, et al. Evaluation of the PFA-100 system in the diagnosis and therapeutic monitoring of patients with von Willebrand disease. Thromb Haemost. 1999;82:35-9.

36. Folsom AR, KK W, Rosamond WD, Sharrett AR, Chambless LE. Prospective study of hemostatic factors and incidence of coronary heart disease: the atherosclerosis risk in communities (ARIC) study. Circulation. 1997;96:1102-8.

37. Montgomery RR, Flood VH. What have we learned from large population studies of von Willebrand disease? Hematology Am Soc Hematol Educ Program. 2016;2016:670-7. https://doi.org/10.1182/asheducation-2016.1.670.

\section{Submit your next manuscript to BioMed Central and we will help you at every step:}

- We accept pre-submission inquiries

- Our selector tool helps you to find the most relevant journal

- We provide round the clock customer support

- Convenient online submission

- Thorough peer review

- Inclusion in PubMed and all major indexing services

- Maximum visibility for your research

Submit your manuscript at www.biomedcentral.com/submit
C Biomed Central 